\title{
An Alternative Procedure for Estimating the Population Mean in Simple Random Sampling
}

\author{
Housila P. Singh \\ School of Studies in Statistics \\ Vikram University, Ujjain \\ India \\ hpsujn@gmail.com \\ Ramkrishna S. Solanki \\ School of Studies in Statistics \\ Vikram University, Ujjain \\ India \\ ramkssolanki@gmail.com
}

\begin{abstract}
This paper deals with the problem of estimating the finite population mean using auxiliary information in simple random sampling. Firstly we have suggested a correction to the mean squared error of the estimator proposed by Gupta and Shabbir (2008). Later we have proposed a ratio type estimator and its properties are studied in simple random sampling. Numerically we have shown that the proposed class of estimators is more efficient than different known estimators including Gupta and Shabbir (2008) estimator.
\end{abstract}

Keywords: Study variate, Auxiliary variate, Finite population mean, Bias, Mean squared error.

\section{Introduction}

Consider a finite population $U=\left(U_{1}, U_{2}, \ldots, U_{N}\right)$ consisting of $N$ units. Let $y$ and $x$ be the auxiliary variables with population means $\bar{Y}$ and $\bar{X}$ respectively. Let a sample of size $n$ be drawn from the population $U$ using simple random sampling without replacement (SRSWOR) scheme. Let $\bar{y}$ and $\bar{x}$ be the sample means of $y$ and $x$ respectively. For estimating the population mean $\bar{Y}$, the usual unbiased estimator, classical ratio and product estimators are respectively defined by

$$
\begin{aligned}
& t_{0}=\bar{y}, \\
& t_{R}=\bar{y} \frac{\bar{x}}{\bar{x}}, \\
& t_{P}=\bar{y} \frac{\bar{x}}{\bar{x}} .
\end{aligned}
$$

Here it is assumed that the population mean $\bar{X}$ of the auxiliary variable $\mathrm{x}$ is known. The classical ratio and product estimators are considered to be practicable in many situations, but they have the limitations of having at the most the same efficiency as that of linear regression estimator. Regression estimator, 
in spite of its lesser practicability, seems to be holding a unique position due to its sound theoretical basis. Some authors including, Jhajj et al. (2006), Kadilar and Cingi (2004, 2006a, b, c), Singh and Espejo (2003, 2007), Upadhyaya and Singh (1999), Singh and Tailor (2003), Singh and Agnihotri (2008), Singh (1986) and the references cited therein, have attempted to formulate the modified estimators in order to provide better alternatives.

Using the transformation

$$
z_{i}=\eta x_{i}+\lambda, i=1,2, \ldots, N \text {. }
$$

on the auxiliary variable x, Gupta and Shabbir (2008) suggested the following ratio type estimator

$$
t_{1}=\left[w_{1} \bar{y}+w_{2}(\bar{X}-\bar{x})\right]\left(\frac{\eta \bar{X}+\lambda}{\eta \bar{x}+\lambda}\right)
$$

for the population mean $\bar{Y}$, where $w_{1}$ and $w_{2}$ are weights whose values are to be determined such that mean squared error of the estimator $t_{1}$ is minimum, and $\eta(\neq 0)$ and $\lambda$ are either constants or functions of the known parameters such as standard deviation $S_{x}$, variance $S_{x}^{2}$, moment ratios $\beta_{1}(x), \beta_{2}(x)$, coefficient of variation $C_{x}$ and correlation coefficient $\rho_{y x}$ between $y$ and $x$ etc.

The variance/MSE of $\bar{y}$ under SRSWOR is given by

$$
\operatorname{MSE}\left(t_{0}=\bar{y}\right)=\frac{(1-f)}{n} \bar{Y}^{2} C_{y}^{2},
$$

where $f=n / N, C_{y}=S_{y} / \bar{Y}$ and $S_{y}^{2}=\sum_{i=1}^{N}\left(y_{i}-\bar{Y}\right)^{2} /(N-1)$.

To the first degree of approximation, the MSEs of classical ratio $t_{R}$ and product $t_{P}$ are respectively given by

$$
\begin{aligned}
& \operatorname{MSE}\left(t_{R}\right)=\frac{(1-f)}{n} \bar{Y}^{2}\left[C_{y}^{2}+C_{x}^{2}(1-2 k)\right] \\
& \operatorname{MSE}\left(t_{P}\right)=\frac{(1-f)}{n} \bar{Y}^{2}\left[C_{y}^{2}+C_{x}^{2}(1+2 k)\right]
\end{aligned}
$$

where

$$
\begin{aligned}
& C_{x}=S_{x} / \bar{X}, k=\rho_{y x}\left(C_{y} / C_{x}\right), \rho_{y x}=\left(S_{y x}\right) /\left(S_{y} S_{x}\right), S_{x}^{2}=\sum_{i=1}^{N}\left(x_{i}-\bar{X}\right)^{2} /(N-1) \quad \text { and } \\
& S_{y x}=\sum_{i=1}^{N}\left(x_{i}-\bar{X}\right)\left(y_{i}-\bar{Y}\right) /(N-1) .
\end{aligned}
$$


To the first degree of approximation, the MSE of the estimator $t_{1}$ is obtained by Gupta and Shabbir (2008) as

$$
\begin{aligned}
& \operatorname{MSE}\left(t_{1}\right) \cong\left(w_{1}-1\right)^{2} \bar{Y}^{2}+\frac{(1-f)}{n}\left[w_{1}^{2} \bar{Y}^{2}\left\{C_{y}^{2}+\tau C_{x}^{2}(\tau-2 k)\right\}\right. \\
& \left.+w_{2}^{2} \bar{X}^{2} C_{x}^{2}-2 w_{1} w_{2} R \bar{X}^{2} C_{x}^{2}(k-\tau)\right],
\end{aligned}
$$

where $R=\bar{Y} / \bar{X}$ and $\tau=\eta \bar{X} /(\eta \bar{X}+\lambda)$.

It is to be noted that the MSE expression obtained by Gupta and Shabbir (2008) is not correct and thus the entire study carried out in the paper by Gupta and Shabbir (2008) are erroneous except concerning the bias. Keeping this in view we have first obtained the correct MSE expression of the estimator $t_{1}$. Later we propose a general class of estimators for population mean $\bar{Y}$ along with its properties. An empirical study is carried out to show the performance of the suggested estimator over others.

\section{MSE expression of Gupta and Shabbir (2008) estimator $t_{1}$}

To obtain the MSE of $t_{1}$ we write

$$
\bar{y}=\bar{Y}\left(1+e_{0}\right), \bar{x}=\bar{X}\left(1+e_{1}\right),
$$

such that

$$
E\left(e_{0}\right)=E\left(e_{1}\right)=0
$$

and

$$
\left.\begin{array}{l}
E\left(e_{0}^{2}\right)=\frac{(1-f)}{n} C_{y}^{2}, \\
E\left(e_{1}^{2}\right)=\frac{(1-f)}{n} C_{x}^{2}, \\
E\left(e_{0} e_{1}\right)=\frac{(1-f)}{n} \rho_{y x} C_{y} C_{x}=\frac{(1-f)}{n} k C_{x}^{2} .
\end{array}\right\}
$$

Expressing $t_{1}$ in terms of e's we have

$$
\mathrm{t}_{1}=\left[\mathrm{w}_{1} \overline{\mathrm{Y}}\left(1+\mathrm{e}_{0}\right)-\mathrm{w}_{2} \overline{\mathrm{X}} \mathrm{e}_{1}\right]\left(1+\tau \mathrm{e}_{1}\right)^{-1} \text {. }
$$

We assume that $\left|\tau \mathrm{e}_{1}\right|<1$ so that the term $\left(1+\tau \mathrm{e}_{1}\right)^{-1}$ is expandable. Expanding the right hand side of (11) we have

$$
\begin{aligned}
& t_{1}=\left[w_{1} \bar{Y}\left(1+e_{0}\right)-w_{2} \bar{X} e_{1}\right]\left[1-\tau e_{1}+\tau^{2} e_{1}^{2}-\ldots\right] \\
& =\left[w_{1} \bar{Y}\left(1+e_{0}\right)-w_{2} \bar{X}_{1}-w_{1} \tau \bar{Y}\left(e_{1}+e_{0} e_{1}\right)+w_{2} \tau \bar{X} e_{1}^{2}\right. \\
& \left.+w_{1} \bar{Y}\left(\tau^{2} e_{1}^{2}+\tau^{2} e_{0} e_{1}^{2}\right)-\ldots\right] .
\end{aligned}
$$


Neglecting terms of e's having power greater than two we have

$$
\mathrm{t}_{1} \cong\left[\mathrm{w}_{1} \overline{\mathrm{Y}}\left(1+\mathrm{e}_{0}\right)-\mathrm{w}_{2} \overline{\mathrm{X}} \mathrm{e}_{1}-\mathrm{w}_{1} \tau \overline{\mathrm{Y}}\left(\mathrm{e}_{1}+\mathrm{e}_{0} \mathrm{e}_{1}\right)+\mathrm{w}_{2} \tau \overline{\mathrm{X}} \mathrm{e}_{1}^{2}+\mathrm{w}_{1} \overline{\mathrm{Y}} \tau^{2} \mathrm{e}_{1}^{2}\right]
$$

Subtracting $\bar{Y}$ from both sides of the above expression, we have

$$
\left(\mathrm{t}_{1}-\overline{\mathrm{Y}}\right) \cong\left[\mathrm{w}_{1} \overline{\mathrm{Y}}\left\{1+\mathrm{e}_{0}-\tau\left(\mathrm{e}_{1}+\mathrm{e}_{0} \mathrm{e}_{1}-\tau \mathrm{e}_{1}^{2}\right)\right\}-\mathrm{w}_{2} \overline{\mathrm{X}}\left(\mathrm{e}_{1}-\tau \mathrm{e}_{1}^{2}\right)-\overline{\mathrm{Y}}\right]
$$

Squaring both sides of (12) and neglecting terms of e's having power greater than two we have

$$
\begin{aligned}
&\left(t_{1}-\bar{Y}\right)^{2} \cong {\left[\bar{Y}^{2}+w_{1}^{2} \bar{Y}^{2}\left\{1+e_{0}^{2}+\tau^{2} e_{1}^{2}+2 e_{0}-2 \tau\left(e_{1}+e_{0} e_{1}-\tau e_{1}^{2}\right)-2 \tau e_{0} e_{1}\right\}\right.} \\
&+w_{2}^{2} \bar{X}^{2} e_{1}^{2}-2 w_{1} \bar{Y}^{2}\left\{1+e_{0}-\tau\left(e_{1}+e_{0} e_{1}-\tau e_{1}^{2}\right)\right\} \\
&\left.+2 w_{2} \bar{Y} \bar{X}\left(e_{1}-\tau e_{1}^{2}\right)-2 w_{1} w_{2} \bar{Y} \bar{X}\left\{\left(e_{1}+e_{0} e_{1}-\tau e_{1}^{2}\right)-\tau e_{1}^{2}\right\}\right]
\end{aligned}
$$

or

$$
\begin{aligned}
\left(t_{1}-\bar{Y}\right)^{2} & =\left[\bar{Y}^{2}+w_{1}^{2} \bar{Y}^{2}\left(1+2 e_{0}-2 \tau e_{1}+e_{0}^{2}+3 \tau^{2} e_{1}^{2}-4 \tau e_{0} e_{1}\right)+w_{2}^{2} \bar{X}^{2} e_{1}^{2}\right. \\
& -2 w_{1} w_{2} \bar{Y} \bar{X}\left(e_{1}+e_{0} e_{1}-2 \tau e_{1}^{2}\right)-2 w_{1} \bar{Y}^{2}\left\{1+e_{0}\right. \\
& \left.\left.-\tau\left(e_{1}+e_{0} e_{1}-\tau e_{1}^{2}\right)\right\}+2 w_{2} \bar{Y} \bar{X}\left(e_{1}-\tau e_{1}^{2}\right)\right] .
\end{aligned}
$$

Taking expectation of both sides of (13) we get the MSE of $t_{1}$ to the first degree of approximation as

$$
\operatorname{MSE}\left(t_{1}\right)=\left[\bar{Y}^{2}+w_{1}^{2} \bar{Y}^{2} \alpha_{1}+w_{2}^{2} \bar{X}^{2} \alpha_{2}-2 w_{1} w_{2} \bar{Y} \bar{X} \alpha_{3}-2 w_{1} \bar{Y}^{2} \alpha_{4}-2 w_{2} \bar{Y} \bar{X} \alpha_{5}\right] \text {, }
$$

where

$$
\begin{aligned}
& \alpha_{1}=\left[1+\frac{(1-f)}{n}\left\{C_{y}^{2}+\tau C_{x}^{2}(3 \tau-4 k)\right\}\right], \\
& \alpha_{2}=\frac{(1-f)}{n} C_{x}^{2}, \\
& \alpha_{3}=\frac{(1-f)}{n} C_{x}^{2}(k-2 \tau), \\
& \alpha_{4}=\left[1-\frac{(1-f)}{n} \tau C_{x}^{2}(k-\tau)\right], \\
& \alpha_{5}=\frac{(1-f)}{n} \tau C_{x}^{2} .
\end{aligned}
$$

Now setting $\frac{\partial \operatorname{MSE}\left(\mathrm{t}_{1}\right)}{\partial \mathrm{w}_{\mathrm{i}}}=0,(\mathrm{i}=1,2)$, we have

$$
\left[\begin{array}{cc}
\bar{Y}^{2} \alpha_{1} & -\alpha_{3} \bar{Y} \bar{X} \\
-\alpha_{3} \bar{Y} \bar{X} & \alpha_{2} \bar{X}^{2}
\end{array}\right]\left[\begin{array}{l}
w_{1} \\
w_{2}
\end{array}\right]=\left[\begin{array}{l}
\bar{Y}^{2} \alpha_{4} \\
\bar{X} \bar{Y} \alpha_{5}
\end{array}\right] \text {. }
$$


Solving (15) we get the optimum values of $w_{1}$ and $w_{2}$ as

$$
\left.\begin{array}{l}
w_{1}=\frac{\left(\alpha_{2} \alpha_{4}+\alpha_{3} \alpha_{5}\right)}{\left(\alpha_{1} \alpha_{2}-\alpha_{3}^{2}\right)}=w_{1}^{*} \\
w_{2}=\frac{R\left(\alpha_{1} \alpha_{5}+\alpha_{3} \alpha_{4}\right)}{\left(\alpha_{1} \alpha_{2}-\alpha_{3}^{2}\right)}=w_{2}^{*}
\end{array}\right\} .
$$

It is assumed that the unknown parameters involved in $\alpha_{i}{ }^{\prime} s(i=1,2,3,4,5)$ and $\mathrm{R}$ are easily estimable from the preliminary data as in Singh and Singh (1984), Tracy and Singh (1997), Tracy et al. (1998), Upadhyaya and Singh (1999), Singh and Vishwakarma (2006), Singh and Espejo (2007), Kadilar and Cingi (2006a) and Singh et al. (2008).

Substitution of (16) in (14) yields the minimum MSE of $t_{1}$ as

$$
M S E_{\min }\left(t_{1}\right)=\bar{Y}^{2}\left[1-\frac{\left(\alpha_{2} \alpha_{4}^{2}+2 \alpha_{3} \alpha_{4} \alpha_{5}+\alpha_{1} \alpha_{5}^{2}\right)}{\left(\alpha_{1} \alpha_{2}-\alpha_{3}^{2}\right)}\right] .
$$

Thus we state the following theorem.

Theorem 1: To the first degree of approximation,

with equality holding if

$$
\operatorname{MSE}\left(t_{1}\right) \geq \bar{Y}^{2}\left[1-\frac{\left(\alpha_{2} \alpha_{4}^{2}+2 \alpha_{3} \alpha_{4} \alpha_{5}+\alpha_{1} \alpha_{5}^{2}\right)}{\left(\alpha_{1} \alpha_{2}-\alpha_{3}^{2}\right)}\right]
$$

$$
\mathrm{w}_{1}=\mathrm{w}_{1}^{*} \text { and } \mathrm{w}_{2}=\mathrm{w}_{2}^{*} \text {. }
$$

Thus the statement "the specific values of $\eta$ and $\lambda$ used in defining various transformations of the auxiliary variable $x$ play no role" given by Gupta and Shabbir (2008, p. 563) is not correct. Expression (17) clearly indicates that there is role of the specific values of $\eta$ and $\lambda$ as minimum MSE of $t_{1}$ depends on $(\eta, \lambda)$ . Thus we provide list of some estimators in Table 1 which are members of the class of estimators $\mathbf{t}_{1}$.

\subsection{Efficiency comparisons}

Kadilar and Cingi (2004) proposed the following class of estimators for population mean $\bar{Y}$ as

$$
t_{k c_{i}}=[\bar{y}+b(\bar{x}-\bar{x})] a_{i}, i=1,2,3,4,5
$$

where $b$ is the sample regression coefficient of $y$ on $x$ and $a_{i}$ 's are defined as

$$
a_{1}=\frac{\bar{x}}{\bar{x}}, a_{2}=\frac{\bar{x}+C_{x}}{\bar{x}+C_{x}}, a_{3}=\frac{\bar{x}+\beta_{2}(x)}{\bar{x}+\beta_{2}(x)}, a_{4}=\frac{\bar{x} \beta_{2}(x)+C_{x}}{\bar{x} \beta_{2}(x)+C_{x}}, a_{5}=\frac{\bar{x} C_{x}+\beta_{2}(x)}{\bar{x} C_{x}+\beta_{2}(x)} \text {. }
$$


To the first degree of approximation, the MSE of $t_{\mathrm{KC}_{i}},(i=1,2,3,4,5)$ are given by

$$
\operatorname{MSE}\left(t_{\mathrm{KC}_{i}}\right)=\frac{(1-f)}{n} \bar{Y}^{2}\left[a_{i}^{*} C_{x}^{2}+C_{y}^{2}\left(1-\rho_{y x}^{2}\right)\right],
$$

where

$$
\begin{aligned}
& a_{1}^{*}=1, a_{2}^{*}=\bar{X} /\left(\bar{X}+C_{x}\right), a_{3}^{*}=\bar{X} /\left(\bar{X}+\beta_{2}(x)\right), a_{4}^{*}=\bar{X} \beta_{2}(x) /\left(\bar{X} \beta_{2}(x)+C_{x}\right) \\
& a_{5}^{*}=\bar{X} C_{x} /\left(\bar{X} C_{x}+\beta_{2}(x)\right) .
\end{aligned}
$$

Kadilar and Cingi (2006c) have suggested another class of estimators for $\bar{Y}$ as

$$
\mathrm{t}_{\mathrm{Kc_{i }}}^{*}=[\overline{\mathrm{y}}+\mathrm{b}(\overline{\mathrm{X}}-\overline{\mathrm{x}})] \mathrm{b}_{\mathrm{i}}, \mathrm{i}=1,2,3,4,5 .
$$

where

$$
\begin{aligned}
& b_{1}=\frac{\bar{X}+\rho_{y x}}{\bar{x}+\rho_{y x}}, b_{2}=\frac{\bar{x} C_{x}+\rho_{y x}}{\bar{x} C_{x}+\rho_{y x}}, b_{3}=\frac{\bar{X} \rho_{y x}+C_{x}}{\bar{x} \rho_{y x}+C_{x}}, b_{4}=\frac{\bar{X} \beta_{2}(x)+\rho_{y x}}{\bar{x} \beta_{2}(x)+\rho_{y x}}, \\
& b_{5}=\frac{\bar{X} \rho_{y x}+\beta_{2}(x)}{\bar{x} \rho_{y x}+\beta_{2}(x)}
\end{aligned}
$$

To the first degree of approximation, the MSE of $\mathrm{t}_{\mathrm{Kc}_{\mathrm{i}}}^{*}(\mathrm{i}=1,2,3,4,5)$ are given by

where

$$
\operatorname{MSE}\left(\mathrm{t}_{\mathrm{kC_{i }}}^{*}\right)=\frac{(1-f)}{n} \bar{Y}^{2}\left[b_{i}^{*} C_{x}^{2}+C_{y}^{2}\left(1-\rho_{\mathrm{yx}}^{2}\right)\right], i=1,2,3,4,5
$$

$$
\begin{aligned}
& b_{1}^{*}=\frac{\bar{X}}{\bar{X}+\rho_{y x}}, b_{2}^{*}=\frac{\bar{X} C_{x}}{\bar{X} C_{x}+\rho_{y x}}, b_{3}^{*}=\frac{\bar{X} \rho_{y x}}{\bar{X} \rho_{y x}+C_{x}}, b_{4}^{*}=\frac{\bar{X} \beta_{2}(x)}{\bar{x} \beta_{2}(x)+\rho_{y x}}, \\
& b_{5}^{*}=\frac{\bar{X} \rho_{y x}}{\bar{X} \rho_{y x}+\beta_{2}(x)} .
\end{aligned}
$$

Kadilar and Cingi (2006a) have further considered the following class of estimators for population mean $\bar{Y}$ as

$$
\mathrm{t}_{\mathrm{Kc_{j }}}^{* *}=\mathrm{k}_{1}[\overline{\mathrm{y}}+\mathrm{b}(\overline{\mathrm{X}}-\overline{\mathrm{x}})] \mathrm{a}_{1}+\mathrm{k}_{2}[\overline{\mathrm{y}}+\mathrm{b}(\overline{\mathrm{X}}-\overline{\mathrm{x}})] \mathrm{a}_{\mathrm{j}}, \mathrm{j}=2,3,4,5
$$

where $k_{1}$ and $k_{2}$ are weight such that $k_{1}+k_{2}=1$.

To the first degree of approximation the common minimum MSE of $\mathrm{t}_{\mathrm{KC}_{\mathrm{j}}}^{* *}$, $(j=1,2,3,4,5)$ is given by

$$
\operatorname{MSE}_{\text {min }}\left(t_{k c_{j}}^{* *}\right)=\frac{(1-f)}{n} \bar{Y}^{2} C_{y}^{2}\left(1-\rho_{y x}^{2}\right),(j=2,3,4,5)
$$




$$
\begin{aligned}
& =\bar{Y}^{2}\left[1+\alpha_{1}-2 \alpha_{4}-\frac{\left(\alpha_{3}+\alpha_{5}\right)^{2}}{\alpha_{2}}\right] \\
& =\operatorname{MSE}\left(t_{\text {Reg }}\right),
\end{aligned}
$$

where $t_{\text {Reg }}=\bar{y}+b(\bar{X}-\bar{x})$ is the usual linear regression estimator of the population mean $\bar{Y}$.

From (6), (17), (19), (21), (23) and (24) we have

$$
\begin{aligned}
& \operatorname{MSE}(\bar{y})-\left\{\operatorname{MSE}\left(t_{\text {Reg }}\right)=\operatorname{MSE}_{\text {min }}\left(t_{\mathrm{Kc}_{j}}^{* *}\right)\right\}=\frac{(1-f)}{n} \bar{Y}^{2} C_{y}^{2} \rho_{\mathrm{yx}}^{2}>0 \text {, } \\
& (j=2,3,4,5) \\
& \operatorname{MSE}\left(\mathrm{t}_{\mathrm{KC}_{\mathrm{i}}}\right)-\left\{\operatorname{MSE}\left(\mathrm{t}_{\mathrm{Reg}}\right)=\operatorname{MSE}_{\min }\left(\mathrm{t}_{\mathrm{KC}_{\mathrm{j}}}^{* *}\right)\right\}=\frac{(1-\mathrm{f})}{\mathrm{n}} \overline{\mathrm{Y}}^{2} \mathrm{C}_{\mathrm{x}}^{2} \mathrm{a}_{\mathrm{i}}^{*}>0 \text {, } \\
& (i=1,2,3,4,5, j=2,3,4,5) \\
& \operatorname{MSE}\left(\mathrm{t}_{\mathrm{KC}_{\mathrm{i}}^{*}}^{*}\right)-\left\{\operatorname{MSE}\left(\mathrm{t}_{\mathrm{Reg}}\right)=\operatorname{MSE}_{\min }\left(\mathrm{t}_{\mathrm{KC}_{\mathrm{j}}}^{* *}\right)\right\}=\frac{(1-\mathrm{f})}{\mathrm{n}} \overline{\mathrm{Y}}^{2} \mathrm{C}_{\mathrm{x}}^{2} \mathrm{~b}_{\mathrm{i}}^{*}>0 \text {, } \\
& \text { ( } i=1,2,3,4,5, j=2,3,4,5) \\
& \left\{\mathrm{MSE}\left(\mathrm{t}_{\mathrm{Reg}}\right)=\mathrm{MSE}_{\text {min }}\left(\mathrm{t}_{\mathrm{KC}_{\mathrm{j}}}^{* *}\right)\right\}-\mathrm{MSE}_{\text {min }}\left(\mathrm{t}_{1}\right) \\
& =\frac{\bar{Y}^{2}\left[\alpha_{2}\left(\alpha_{1}-\alpha_{4}\right)-\alpha_{3}\left(\alpha_{3}+\alpha_{5}\right)\right]^{2}}{\alpha_{2}\left(\alpha_{1} \alpha_{2}-\alpha_{3}^{2}\right)}>0,(j=2,3,4,5)
\end{aligned}
$$

From (25), (26), (27) and (28) we have the following inequalities,

$$
\begin{aligned}
& \left\{\operatorname{MSE}\left(\mathrm{t}_{\text {Reg }}\right)=\operatorname{MSE}_{\text {min }}\left(\mathrm{t}_{\mathrm{Kc}}^{* *}\right)<\operatorname{MSE}(\bar{y}),(j=2,3,4,5)\right. \\
& \left\{\operatorname{MSE}\left(t_{\text {Reg }}\right)=\operatorname{MSE}_{\text {min }}\left(t_{K_{c_{j}}}^{* *}\right)<\operatorname{MSE}\left(t_{K_{i}}\right),(i=1,2,3,4,5, j=2,3,4,5)\right. \\
& \left\{\operatorname{MSE}\left(t_{\text {Reg }}\right)=\operatorname{MSE}_{\text {min }}\left(t_{K_{C_{j}}}^{* *}\right)<\operatorname{MSE}\left(t_{K_{C_{i}}^{*}}^{*}\right),(i=1,2,3,4,5, j=2,3,4,5)\right. \\
& \operatorname{MSE}_{\text {min }}\left(t_{1}\right)<\left\{\operatorname{MSE}\left(t_{\text {Reg }}\right)=\operatorname{MSE}_{\text {min }}\left(t_{\mathrm{KC}_{j}}^{* *}\right)\right\},(j=2,3,4,5)
\end{aligned}
$$

From (29), (30), (31) and (32) we have

$$
\begin{aligned}
& \operatorname{MSE}_{\text {min }}\left(\mathrm{t}_{1}\right)<\left\{\operatorname{MSE}\left(\mathrm{t}_{\mathrm{Reg}}\right)=\operatorname{MSE}_{\text {min }}\left(\mathrm{t}_{\mathrm{KC}_{\mathrm{j}}}^{* *}\right)\right\}<\operatorname{MSE}(\overline{\mathrm{y}}),(\mathrm{j}=2,3,4,5) \\
& \operatorname{MSE}_{\text {min }}\left(\mathrm{t}_{1}\right)<\left\{\operatorname{MSE}\left(\mathrm{t}_{\mathrm{Reg}}\right)=\operatorname{MSE}_{\text {min }}\left(\mathrm{t}_{\mathrm{KC}_{\mathrm{j}}}^{* *}\right)\right\}<\operatorname{MSE}\left(\mathrm{t}_{\mathrm{KC}_{\mathrm{j}}}\right) \text {, } \\
& \text { ( } i=1,2,3,4,5, j=2,3,4,5) \\
& \operatorname{MSE}_{\text {min }}\left(\mathrm{t}_{1}\right)<\left\{\operatorname{MSE}\left(\mathrm{t}_{\mathrm{Reg}}\right)=\operatorname{MSE}_{\text {min }}\left(\mathrm{t}_{\mathrm{KC}_{\mathrm{j}}}^{* *}\right)\right\}<\operatorname{MSE}\left(\mathrm{t}_{\mathrm{KC}_{\mathrm{j}}}^{*}\right) \text {, } \\
& \text { ( } i=1,2,3,4,5, j=2.3 .4,5)
\end{aligned}
$$


It follows from (33), (34) and (35) that the class of estimators $t_{1}$ due to Gupta and Shabbir (2008) is better than usual unbiased estimator $\bar{y}$, usual linear regression estimator $t_{\text {Reg }}$ [and hence the usual ratio $\left(t_{R}\right)$ and product $\left(t_{P}\right)$ estimators] and the estimators due to Kadilar and Cingi (2004, 2006a, c).

\section{A general class of estimators}

We define a class of estimators for population mean $\bar{Y}$ as

$$
\mathrm{t}_{2}=\psi_{1} \overline{\mathrm{y}}\left(\frac{\eta \overline{\mathrm{X}}+\lambda}{\eta \overline{\mathrm{x}}+\lambda}\right)+\psi_{2}(\overline{\mathrm{X}}-\overline{\mathrm{x}})\left(\frac{\eta \overline{\mathrm{X}}+\lambda}{\eta \overline{\mathrm{X}}+\lambda}\right)^{2},
$$

where $\psi_{1}$ and $\psi_{2}$ are suitably chosen constants such that MSE of $t_{2}$ is minimum and $(\eta, \lambda)$ are same as defined earlier. A large number of estimators can be generated from the suggested estimator $t_{2}$ for suitable values of $\left(\psi_{1}, \psi_{2}, \eta, \lambda\right)$.

Expressing $t_{2}$ in terms of e's we have

$$
\mathrm{t}_{2}=\psi_{1} \overline{\mathrm{Y}}\left(1+\mathrm{e}_{0}\right)\left(1+\tau \mathrm{e}_{1}\right)^{-1}-\psi_{2} \overline{\mathrm{X}} \mathrm{e}_{1}\left(1+\tau \mathrm{e}_{1}\right)^{-2}
$$

We assume that $\left|\tau e_{1}\right|<1$, so that $\left(1+\tau e_{1}\right)^{-1}$ and $\left(1+\tau e_{1}\right)^{-2}$ are expandable. Expanding the right hand side of (37), multiplying out and neglecting terms of e's having power greater than two we have

$$
\begin{aligned}
& \mathrm{t}_{2} \cong \psi_{1} \overline{\mathrm{Y}}\left[1+\mathrm{e}_{0}-\tau \mathrm{e}_{1}-\tau \mathrm{e}_{0} \mathrm{e}_{1}+\tau^{2} \mathrm{e}_{1}^{2}\right]-\psi_{2} \overline{\mathrm{X}}\left[\mathrm{e}_{1}-2 \tau \mathrm{e}_{1}^{2}\right] \\
& \text { or } \quad\left(\mathrm{t}_{2}-\overline{\mathrm{Y}}\right) \cong \overline{\mathrm{Y}}\left[\psi_{1}\left\{1+\mathrm{e}_{0}-\tau \mathrm{e}_{1}-\tau \mathrm{e}_{0} \mathrm{e}_{1}+\tau^{2} \mathrm{e}_{1}^{2}\right\}-\psi_{2}\left(\frac{1}{\mathrm{R}}\right)\left(\mathrm{e}_{1}-2 \tau \mathrm{e}_{1}^{2}\right)-1\right]
\end{aligned}
$$

Taking expectation of both sides of (38) we get the bias of $t_{2}$ to the first degree of approximation as

$$
\begin{aligned}
& B\left(t_{2}\right)=\bar{Y}\left[\psi_{1}\left\{1+\frac{(1-f)}{n} \tau(\tau-k) C_{x}^{2}\right\}+2 \psi_{2}\left(\frac{1}{R}\right) \frac{(1-f)}{n} \tau C_{x}^{2}-1\right] \\
& =\bar{Y}\left(\psi_{1}-1\right)+\frac{(1-f)}{n} \tau C_{x}^{2}\left[\psi_{1}\{\tau-k\}+2 \psi_{2}\left(\frac{1}{R}\right)\right] .
\end{aligned}
$$

Squaring both sides of (38) and neglecting terms of e's having power greater than two we have

$$
\begin{aligned}
& \left(t_{2}-\bar{Y}\right)^{2}=\bar{Y}^{2}\left[\psi_{1}^{2}\left\{1+2 e_{0}-2 \tau e_{1}+e_{0}^{2}-4 \tau e_{0} e_{1}+3 \tau^{2} e_{1}^{2}\right\}+\psi_{2}^{2}\left(\frac{1}{R^{2}}\right) e_{1}^{2}\right. \\
& -2 \psi_{1} \psi_{2}\left(\frac{1}{R}\right)\left\{e_{1}+e_{0} e_{1}-3 \tau e_{1}^{2}\right\}-2 \psi_{1}\left\{1+e_{0}-\tau e_{1}-\tau e_{0} e_{1}\right.
\end{aligned}
$$




$$
\left.\left.+\tau^{2} e_{1}^{2}\right\}+2 \psi_{2}\left(\frac{1}{R}\right)\left(e_{1}-2 \tau e_{1}^{2}\right)+1\right]
$$

Taking expectation of both sides of (40) we get the MSE of $t_{2}$ to the first degree of approximation as

where

$$
\operatorname{MSE}\left(t_{2}\right)=\bar{Y}^{2}\left[1+\psi_{1}^{2} A+\psi_{2}^{2}\left(\frac{1}{R^{2}}\right) B+2 \psi_{1} \psi_{2}\left(\frac{1}{R}\right) C-2 \psi_{1} D-2 \psi_{2}\left(\frac{1}{R}\right) E\right],
$$

$$
\begin{aligned}
& A=\left[1+\frac{(1-f)}{n}\left\{C_{y}^{2}+\tau C_{x}^{2}(3 \tau-4 k)\right\}\right] \\
& B=\frac{(1-f)}{n} C_{x}^{2} \\
& C=\frac{(1-f)}{n} C_{x}^{2}(3 \tau-k), \\
& D=\left[1+\frac{(1-f)}{n} \tau C_{x}^{2}(\tau-k)\right], \\
& E=\frac{2(1-f)}{n} \tau C_{x}^{2} .
\end{aligned}
$$

Differentiating (41) with respect to $\psi_{1}$ and $\psi_{2}$ partially and equating to zero we get

$$
\left[\begin{array}{cc}
\mathrm{A} & \left(\frac{1}{\mathrm{R}}\right) \mathrm{C} \\
\left(\frac{1}{\mathrm{R}}\right) \mathrm{C} & \left(\frac{1}{\mathrm{R}^{2}}\right) \mathrm{B}
\end{array}\right]\left[\begin{array}{l}
\psi_{1} \\
\psi_{2}
\end{array}\right]=\left[\begin{array}{c}
\mathrm{D} \\
1 \\
\left(\frac{1}{\mathrm{R}}\right) \mathrm{E}
\end{array}\right]
$$

Solving (42) we get the optimum values of $\psi_{1}$ and $\psi_{2}$ as

$$
\left.\begin{array}{l}
\psi_{1}=\frac{(B D-C E)}{\left(A B-C^{2}\right)}=\psi_{1}^{*} \text { (say) } \\
\psi_{2}=\frac{(A E-C D)}{\left(A B-C^{2}\right)}=\psi_{2}^{*} \text { (say) }
\end{array}\right\} .
$$

Thus the resulting minimum MSE of $t_{2}$ is given by

$$
\operatorname{MSE}_{\text {min }}\left(t_{2}\right)=\bar{Y}^{2}\left[1-\frac{\left(B D^{2}-2 C D E+A E^{2}\right)}{\left(A B-C^{2}\right)}\right] \text {. }
$$

Now we established the following theorem. 
Theorem 2: To the first degree of approximation,

$\operatorname{MSE}\left(\mathrm{t}_{2}\right) \geq \overline{\mathrm{Y}}^{2}\left[1-\frac{\left(\mathrm{BD}^{2}-2 \mathrm{CDE}+\mathrm{AE}^{2}\right)}{\left(\mathrm{AB}-\mathrm{C}^{2}\right)}\right]$

with equality holding if

$\psi_{1}=\psi_{1}^{*}$ and $\psi_{2}=\psi_{2}^{*}$.

\subsection{Particular case $\left(\psi_{1}=1\right)$}

Putting $\psi_{1}=1$ in (36), we get an estimator for $\bar{Y}$ as

$$
\mathrm{t}_{2}^{*}=\overline{\mathrm{y}}\left(\frac{\eta \overline{\mathrm{X}}+\lambda}{\eta \overline{\mathrm{x}}+\lambda}\right)+\psi_{2}(\overline{\mathrm{X}}-\overline{\mathrm{x}})\left(\frac{\eta \overline{\mathrm{X}}+\lambda}{\eta \overline{\mathrm{x}}+\lambda}\right)^{2} .
$$

To the first degree of approximation, the bias and MSE of $t_{2}^{*}$ are respectively obtained by putting $\psi_{1}=1$ in (39) and (41) as

$$
\begin{aligned}
& B\left(t_{2}^{*}\right)=\frac{(1-f)}{n} \tau C_{x}^{2}\left[(\tau-k)+2 \psi_{2}\left(\frac{1}{R}\right)\right], \\
& \operatorname{MSE}\left(t_{2}^{*}\right)=\bar{Y}^{2}\left[1+A-2 D+\psi_{2}^{2}\left(\frac{1}{R^{2}}\right) B+2 \psi_{2}\left(\frac{1}{R}\right)(C-E)\right] .
\end{aligned}
$$

The $\operatorname{MSE}\left(\mathrm{t}_{2}^{*}\right)$ at $(47)$ is minimized for

$$
\psi_{2}=\frac{(\mathrm{C}-\mathrm{E}) \mathrm{R}}{\mathrm{B}}=\psi_{20} \text { (say). }
$$

Thus the resulting minimum MSE of $t_{2}^{*}$ is given by

$$
\operatorname{MSE}_{\text {min }}\left(t_{2}^{*}\right)=\bar{Y}^{2}\left[1+A-2 D-\frac{(C-E)^{2}}{B}\right]=\frac{(1-f)}{n} S_{y}^{2}\left(1-\rho^{2}\right) \text {, }
$$

which equals to the approximate variance of the usual regression estimator $\mathrm{t}_{\text {Reg }}=\overline{\mathrm{y}}+\mathrm{b}(\overline{\mathrm{X}}-\overline{\mathrm{x}})$.

Thus we established the following theorem.

Theorem 3: To the first degree of approximation,

$$
\operatorname{MSE}\left(t_{2}^{*}\right) \geq \bar{Y}^{2}\left[(1+A-2 D)-\frac{(C-E)^{2}}{B}\right]
$$

with equality holding if

$$
\psi_{2}=\psi_{20} .
$$


From (44) and (49) we have

$$
\operatorname{MSE}_{\text {min }}\left(t_{2}^{*}\right)-\operatorname{MSE}_{\text {min }}\left(t_{2}\right)=\frac{\bar{Y}^{2}[B(A-D)-C(C-E)]^{2}}{\left(A B-C^{2}\right)}>0 \text {, }
$$

which clearly shows that the proposed class of estimators $t_{2}$ is better than $t_{2}^{*}$ (or usual linear regression estimator $t_{\text {Reg }}$ ) and hence the usual unbiased estimator $\bar{y}$ , ratio estimator $t_{R}$, product estimator $t_{P}$ and the estimators due to Kadilar and Cingi (2004, 2006 a, c).

Remark 3.1: It is observed from the expressions in (43) and (48) that the proposed classes of estimators $t_{1}$ and $t_{2}^{*}$ will attained their minimum mean squared errors respectively in (44) and (49) only when the unknown population parameters $C_{y}, C_{x}, \beta_{1}(x), \beta_{2}(x), k, \rho_{y x}$ and $S_{x}$ are known. To use such estimators in practice one has to use some guessed values of $C_{y}, C_{x}, \beta_{1}(x)$, $\beta_{2}(x), k, \rho_{y x}$ and $S_{x}$, either through past experience or through a pilot sample survey [see Srivastava and Jhajj (1980, p. 92)]. Das and Tripathi (1978, sec. 3) have illustrated that even if the values of the constants used in the estimators are not exactly equal to their optimum values as given by (43) and (48) but are close enough, the resulting estimators will be better than the usual unbiased estimator $\bar{y}$. For more detailed discussion on this issue, the reader is referred to Reddy (1973, 1974), Sahai and Ray (1980), Ray and Sahai (1980), Prasad (1989), Lui (1990), Prasad and Singh (1990, 1992) and Ahmed et al. (2000, 2003). On the otherhand, following Srivastava and Jhajj (1983) it can be also shown that the estimator $t_{2}$ and $t_{2}^{*}$ with estimated optimum values obtained by their consistent estimators, attain the same minimum mean squared errors of estimators $t_{2}$ and $t_{2}^{*}$ based on optimum values, up to the first order of approximation [see, Jhajj et al. (2005, p. 28)].

Now we provide list of some ratio-type estimators in Table 2, which are members of the proposed estimator $t_{2}$. A list of some known estimators are also given in Table 3 , which are members of the estimators $t_{1}$ and $t_{2}$. 
Table 1: Some ratio-type estimators which are members of $t_{1}$.

\begin{tabular}{|c|c|c|}
\hline \multirow{2}{*}{ Estimators } & \multicolumn{2}{|c|}{ Values of constants $(\eta, \lambda)$} \\
\hline & $\eta$ & $\lambda$ \\
\hline $\mathrm{t}_{1(1)}^{\mathrm{r}}=\left\{\mathrm{w}_{1} \overline{\mathrm{y}}+\mathrm{w}_{2}(\overline{\mathrm{X}}-\overline{\mathrm{x}})\right\}\left(\frac{\mathrm{C}_{\mathrm{x}} \overline{\mathrm{X}}+1}{\mathrm{C}_{\mathrm{x}} \overline{\mathrm{x}}+1}\right)$ & $C_{x}$ & 1 \\
\hline$t_{1(2)}^{r}=\left\{w_{1} \bar{y}+w_{2}(\bar{X}-\bar{x})\right\}\left\{\frac{\beta_{2}(x) \bar{X}+1}{\beta_{2}(x) \bar{x}+1}\right\}$ & $\beta_{2}(x)$ & 1 \\
\hline $\mathrm{t}_{1(3)}^{\mathrm{r}}=\left\{\mathrm{w}_{1} \overline{\mathrm{y}}+\mathrm{w}_{2}(\overline{\mathrm{X}}-\overline{\mathrm{x}})\right\}\left\{\frac{\beta_{2}(\mathrm{x}) \overline{\mathrm{X}}+\mathrm{C}_{\mathrm{x}}}{\beta_{2}(\mathrm{x}) \overline{\mathrm{x}}+\mathrm{C}_{\mathrm{x}}}\right\}$ & $\beta_{2}(x)$ & $C_{x}$ \\
\hline$t_{1(4)}^{r}=\left\{w_{1} \bar{y}+w_{2}(\bar{X}-\bar{x})\right\}\left(\frac{\bar{X}+\rho_{y x}}{\bar{x}+\rho_{y x}}\right)$ & 1 & $\rho_{y x}$ \\
\hline$t_{1(5)}^{r}=\left\{w_{1} \bar{y}+w_{2}(\bar{X}-\bar{x})\right\}\left(\frac{C_{x} \bar{X}+\rho_{y x}}{C_{x} \bar{x}+\rho_{y x}}\right)$ & $C_{x}$ & $\rho_{\mathrm{yx}}$ \\
\hline $\mathrm{t}_{1(6)}^{\mathrm{r}}=\left\{\mathrm{w}_{1} \overline{\mathrm{y}}+\mathrm{w}_{2}(\overline{\mathrm{X}}-\overline{\mathrm{x}})\right\}\left\{\frac{\beta_{2}(\mathrm{x}) \overline{\mathrm{X}}+\rho_{\mathrm{yx}}}{\beta_{2}(\mathrm{x}) \overline{\mathrm{x}}+\rho_{\mathrm{yx}}}\right\}$ & $\beta_{2}(x)$ & $\rho_{\mathrm{yx}}$ \\
\hline$t_{1(7)}^{r}=\left\{w_{1} \bar{y}+w_{2}(\bar{X}-\bar{x})\right\}\left(\frac{S_{x} \bar{X}+1}{S_{x} \bar{x}+1}\right)$ & $S_{x}$ & 1 \\
\hline $\mathrm{t}_{1(8)}^{\mathrm{r}}=\left\{\mathrm{w}_{1} \overline{\mathrm{y}}+\mathrm{w}_{2}(\overline{\mathrm{X}}-\overline{\mathrm{x}})\right\}\left\{\frac{\beta_{2}(\mathrm{x}) \overline{\mathrm{X}}+\mathrm{S}_{\mathrm{x}}}{\beta_{2}(\mathrm{x}) \overline{\mathrm{x}}+\mathrm{S}_{\mathrm{x}}}\right\}$ & $\beta_{2}(x)$ & $S_{x}$ \\
\hline$t_{1(9)}^{r}=\left\{w_{1} \bar{y}+w_{2}(\bar{X}-\bar{x})\right\}\left(\frac{S_{x} \bar{X}+\rho_{y x}}{S_{x} \bar{x}+\rho_{y x}}\right)$ & $S_{x}$ & $\rho_{\mathrm{yx}}$ \\
\hline $\mathrm{t}_{1(10)}^{\mathrm{r}}=\left\{\mathrm{w}_{1} \overline{\mathrm{y}}+\mathrm{w}_{2}(\overline{\mathrm{X}}-\overline{\mathrm{x}})\right\}\left(\frac{\overline{\mathrm{x}}}{\overline{\mathrm{x}}}\right)$ & 1 & 0 \\
\hline
\end{tabular}


Table 2: Some ratio-type estimators which are members of $t_{2}$.

\begin{tabular}{|c|c|c|}
\hline \multirow{2}{*}{ Estimators } & \multicolumn{2}{|c|}{ Values of constants $(\eta, \lambda)$} \\
\hline & $\eta$ & $\lambda$ \\
\hline$t_{2(1)}^{r}=\psi_{1} \bar{y}\left(\frac{C_{x} \bar{X}+1}{C_{x} \bar{x}+1}\right)+\psi_{2}(\bar{X}-\bar{x})\left(\frac{C_{x} \bar{X}+1}{C_{x} \bar{x}+1}\right)^{2}$ & $C_{x}$ & 1 \\
\hline $\mathrm{t}_{2(2)}^{\mathrm{r}}=\psi_{1} \overline{\mathrm{y}}\left\{\frac{\beta_{2}(\mathrm{x}) \overline{\mathrm{X}}+1}{\beta_{2}(\mathrm{x}) \overline{\mathrm{x}}+1}\right\}+\psi_{2}(\overline{\mathrm{X}}-\overline{\mathrm{x}})\left\{\frac{\beta_{2}(\mathrm{x}) \overline{\mathrm{X}}+1}{\beta_{2}(\mathrm{x}) \overline{\mathrm{x}}+1}\right\}^{2}$ & $\beta_{2}(x) 1$ & 1 \\
\hline $\mathrm{t}_{2(3)}^{\mathrm{r}}=\psi_{1} \overline{\mathrm{y}}\left\{\frac{\beta_{2}(\mathrm{x}) \overline{\mathrm{X}}+\mathrm{C}_{\mathrm{x}}}{\beta_{2}(\mathrm{x}) \overline{\mathrm{x}}+\mathrm{C}_{\mathrm{x}}}\right\}+\psi_{2}(\overline{\mathrm{X}}-\overline{\mathrm{x}})\left\{\frac{\beta_{2}(\mathrm{x}) \overline{\mathrm{X}}+\mathrm{C}_{\mathrm{x}}}{\beta_{2}(\mathrm{x}) \overline{\mathrm{x}}+\mathrm{C}_{\mathrm{x}}}\right\}^{2}$ & $\beta_{2}(x)$ & $C_{x}$ \\
\hline $\mathrm{t}_{2(4)}^{\mathrm{r}}=\psi_{1} \overline{\mathrm{y}}\left(\frac{\overline{\mathrm{X}}+\rho_{\mathrm{yx}}}{\overline{\mathrm{X}}+\rho_{\mathrm{yx}}}\right)+\psi_{2}(\overline{\mathrm{X}}-\overline{\mathrm{x}})\left(\frac{\overline{\mathrm{X}}+\rho_{\mathrm{yx}}}{\overline{\mathrm{X}}+\rho_{\mathrm{yx}}}\right)^{2}$ & 1 & $\rho_{y x}$ \\
\hline$t_{2(5)}^{r}=\psi_{1} \bar{y}\left(\frac{C_{x} \bar{X}+\rho_{y x}}{C_{x} \bar{x}+\rho_{y x}}\right)+\psi_{2}(\bar{X}-\bar{x})\left(\frac{C_{x} \bar{X}+\rho_{y x}}{C_{x} \bar{x}+\rho_{y x}}\right)^{2}$ & $C_{x}$ & $\rho_{y x}$ \\
\hline $\mathrm{t}_{2(6)}^{\mathrm{r}}=\psi_{1} \overline{\mathrm{y}}\left\{\frac{\beta_{2}(\mathrm{x}) \overline{\mathrm{X}}+\rho_{\mathrm{yx}}}{\beta_{2}(\mathrm{x}) \overline{\mathrm{x}}+\rho_{\mathrm{yx}}}\right\}+\psi_{2}(\overline{\mathrm{X}}-\overline{\mathrm{x}})\left\{\frac{\beta_{2}(\mathrm{x}) \overline{\mathrm{X}}+\rho_{\mathrm{yx}}}{\beta_{2}(\mathrm{x}) \overline{\mathrm{X}}+\rho_{\mathrm{yx}}}\right\}^{2}$ & $\beta_{2}(x)$ & $\rho_{y x}$ \\
\hline $\mathrm{t}_{2(7)}^{\mathrm{r}}=\psi_{1} \overline{\mathrm{y}}\left(\frac{\mathrm{S}_{\mathrm{x}} \overline{\mathrm{X}}+1}{\mathrm{~S}_{\mathrm{x}} \overline{\mathrm{x}}+1}\right)+\psi_{2}(\overline{\mathrm{X}}-\overline{\mathrm{x}})\left(\frac{\mathrm{S}_{\mathrm{x}} \overline{\mathrm{X}}+1}{\mathrm{~S}_{\mathrm{x}} \overline{\mathrm{X}}+1}\right)^{2}$ & $S_{x}$ & 1 \\
\hline $\mathrm{t}_{2(8)}^{\mathrm{r}}=\psi_{1} \overline{\mathrm{y}}\left\{\frac{\beta_{2}(\mathrm{x}) \overline{\mathrm{X}}+\mathrm{S}_{\mathrm{x}}}{\beta_{2}(\mathrm{x}) \overline{\mathrm{x}}+\mathrm{S}_{\mathrm{x}}}\right\}+\psi_{2}(\overline{\mathrm{X}}-\overline{\mathrm{x}})\left\{\frac{\beta_{2}(\mathrm{x}) \overline{\mathrm{X}}+\mathrm{S}_{\mathrm{x}}}{\beta_{2}(\mathrm{x}) \overline{\mathrm{x}}+\mathrm{S}_{\mathrm{x}}}\right\}^{2}$ & $\beta_{2}(x)$ & $S_{x}$ \\
\hline$t_{2(9)}^{r}=\psi_{1} \bar{y}\left(\frac{S_{x} \bar{X}+\rho_{y x}}{S_{x} \bar{X}+\rho_{y x}}\right)+\psi_{2}(\bar{X}-\bar{x})\left(\frac{S_{x} \bar{X}+\rho_{y x}}{S_{x} \bar{X}+\rho_{y x}}\right)^{2}$ & $S_{x}$ & $\rho_{y x}$ \\
\hline$t_{2(10)}^{r}=\psi_{1} \bar{y}\left(\frac{\bar{X}}{\bar{x}}\right)+\psi_{2}(\bar{X}-\bar{x})\left(\frac{\bar{X}}{\bar{x}}\right)^{2}$ & 1 & 0 \\
\hline
\end{tabular}


Table 3: Some known estimators which are members of the estimators $t_{1}$ and $\mathrm{t}_{2}$

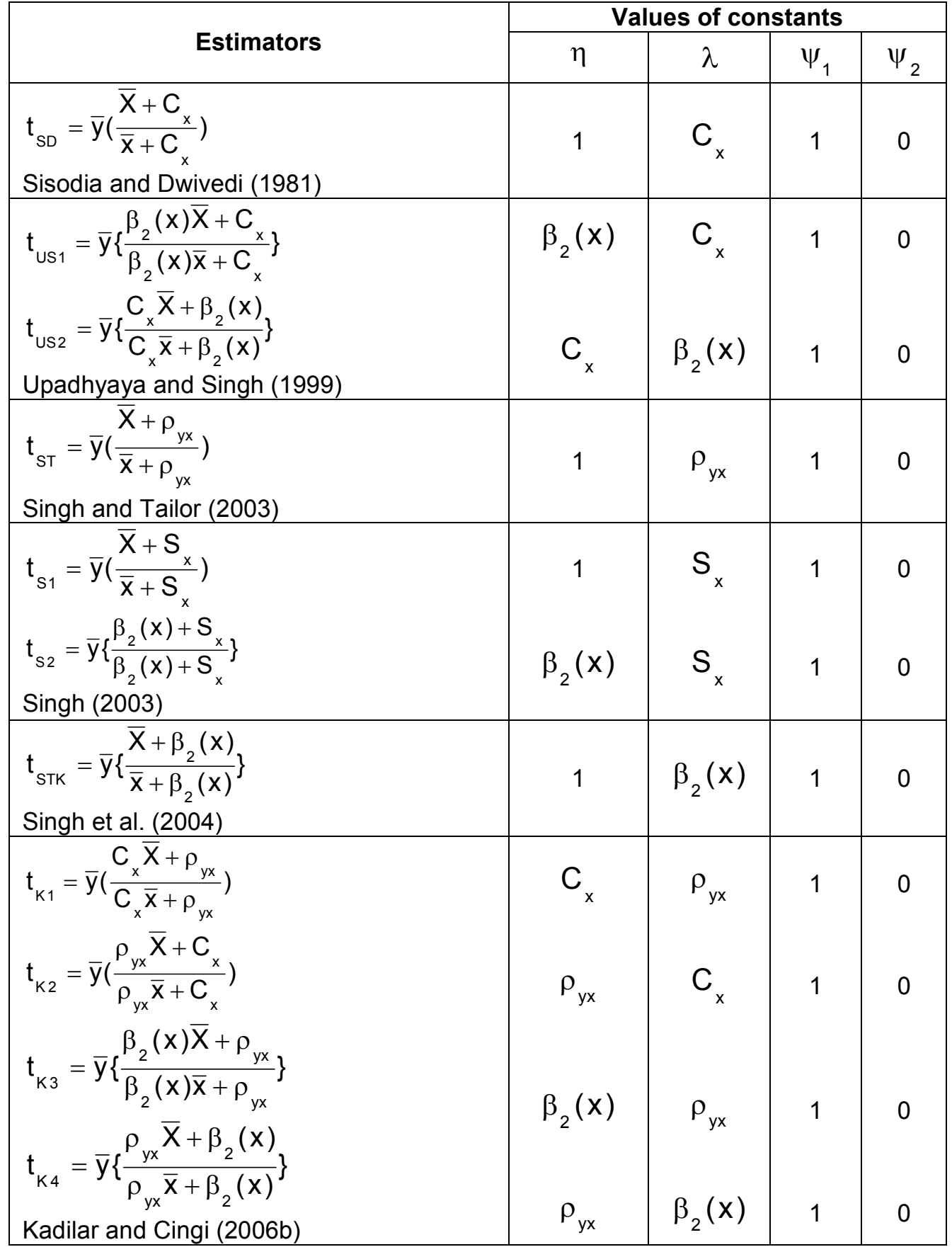

\section{Empirical study}

In this section, we evaluate the performances of various estimators using following data sets which arepreviously used in the literature.

Population 1: [Source: Kadilar and Cingi (2006c)]

$N=200, n=50, \bar{Y}=500, \bar{X}=25, C_{y}=15, C_{x}=2, \rho_{y x}=0.90, \beta_{2}(x)=50$. 
Population 2: [Source: Kadilar and Cingi(2004), Kadilar and Cingi (2006a)]

$N=106, n=20, \bar{Y}=2212.59, \bar{X}=27421.70, C_{y}=5.22$,

$C_{x}=2.10, \rho_{y x}=0.86, \beta_{2}(x)=34.57$.

Population 3: [Source: Kadilar and Cingi (2006b)]

$\mathrm{N}=104, \mathrm{n}=20, \overline{\mathrm{Y}}=625.37, \bar{X}=13.93, \mathrm{C}_{\mathrm{y}}=1.866, \mathrm{C}_{\mathrm{x}}=1.653$,

$\rho_{\mathrm{yx}}=0.865, \beta_{2}(x)=17.516$.

Table 4: MSEs and PREs of different known estimators

\begin{tabular}{|c|c|c|c|c|c|c|}
\hline \multirow{2}{*}{ Estimators } & \multicolumn{2}{|c|}{ Population 1} & \multicolumn{2}{|c|}{ Population 2} & \multicolumn{2}{|c|}{ Population 3} \\
\hline & MSE & PRE & MSE & PRE & MSE & PRE \\
\hline$t_{0}=\bar{y}$ & 843750.00 & 100.000 & 5411348.28 & 100.000 & 54993.75 & 100.000 \\
\hline$t_{R}$ & 656250.00 & 128.571 & 2542740.30 & 212.816 & 13869.96 & 396.495 \\
\hline$t_{S D}$ & 669110.08 & 126.100 & 2542892.90 & 212.803 & 14140.05 & 388.922 \\
\hline $\mathrm{t}_{\text {us1 }}$ & 656525.60 & 128.517 & 2542744.71 & 212.815 & 13858.25 & 396.831 \\
\hline $\mathrm{t}_{\text {us2 }}$ & 746250.00 & 113.065 & 2543936.23 & 212.716 & 21047.63 & 261.282 \\
\hline $\mathrm{t}_{\mathrm{ST}}$ & 662262.32 & 127.404 & 2542802.79 & 212.810 & 13898.70 & 395.676 \\
\hline$t_{s 1}$ & 777916.67 & 108.463 & 4294609.80 & 126.003 & 29357.64 & 187.323 \\
\hline$t_{\mathrm{s} 2}$ & 662906.80 & 127.280 & 2659735.92 & 203.454 & 14015.67 & 392.373 \\
\hline $\mathrm{t}_{\mathrm{KC} 2}$ & 173172.58 & 487.231 & 2284777.20 & 236.844 & 48331.50 & 113.784 \\
\hline $\mathrm{t}_{\mathrm{KC} 3}$ & 161979.17 & 520.900 & 2282707.29 & 237.058 & 22314.56 & 246.448 \\
\hline $\mathrm{t}_{\mathrm{KC} 4}$ & 175264.61 & 481.415 & 2284907.44 & 236.830 & 56422.67 & 97.467 \\
\hline $\mathrm{t}_{\mathrm{KC} 5}$ & 164062.50 & 514.286 & 2283860.74 & 236.939 & 27766.95 & 198.055 \\
\hline$t_{K 1}$ & 659304.79 & 127.976 & 2542770.06 & 212.813 & 13852.97 & 396.982 \\
\hline $\mathrm{t}_{\mathrm{K} 2}$ & 670431.59 & 125.852 & 2542917.74 & 212.801 & 14252.91 & 385.842 \\
\hline$t_{K 3}$ & 656374.12 & 128.547 & 2542742.10 & 212.815 & 13863.32 & 396.685 \\
\hline $\mathrm{t}_{\mathrm{K} 4}$ & 782349.88 & 107.848 & 2545659.17 & 212.572 & 27813.65 & 197.722 \\
\hline$t_{\mathrm{KC} 1}^{*}$ & 174288.14 & 484.112 & 2284856.39 & 236.835 & 52102.80 & 105.549 \\
\hline$t_{\mathrm{KC} 2}^{*}$ & 174786.74 & 482.731 & 2284885.16 & 236.832 & 53933.14 & 101.967 \\
\hline$t_{\mathrm{KC} 3}^{*}$ & 172963.48 & 487.820 & 2284755.36 & 236.846 & 47217.40 & 116.469 \\
\hline $\mathrm{t}_{\mathrm{KC} 4}^{*}$ & 175290.92 & 481.343 & 2284909.73 & 236.830 & 56697.15 & 96.996 \\
\hline$t_{\mathrm{KC} 5}^{*}$ & 161757.21 & 521.615 & 2282349.28 & 237.096 & 21014.10 & 261.699 \\
\hline$t_{K C j}^{* *}$ or $t_{R e g}$ & 160312.50 & 526.316 & 1409115.09 & 384.025 & 13846.05 & 397.180 \\
\hline
\end{tabular}


Table 5: Corrected optimum values $\left(w_{1}^{*}, w_{2}^{*}\right)$, MSEs and PREs of different estimators $\left(\mathrm{t}_{1(\mathrm{i})}^{\mathrm{r}}, \mathrm{i}=1\right.$ to 10) generated from Gupta and Shabbir (2008) estimator $t_{1}$.

\begin{tabular}{|c|c|c|c|c|c|c|c|c|c|c|c|c|}
\hline \multirow[b]{2}{*}{ Estimators } & \multicolumn{4}{|c|}{ Population 1} & \multicolumn{4}{|c|}{ Population 2} & \multicolumn{4}{|c|}{ Population 3} \\
\hline & $\mathrm{w}_{1}^{*}$ & $w_{2}^{*}$ & MSE & PRE & $\mathrm{w}_{1}^{*}$ & $\mathrm{~W}_{2}^{*}$ & MSE & PRE & $\mathrm{w}_{1}^{*}$ & $w_{2}^{*}$ & MSE & PRE \\
\hline$t_{1(1)}^{r}$ & 0.60 & 76.61 & 95396.05 & 884.471 & 0.74 & 0.09 & 1043368.08 & 518.642 & 0.96 & 2.41 & 13321.23 & 412.828 \\
\hline$t_{1(2)}^{r}$ & 0.59 & 76.48 & 95304.34 & 885.322 & 0.74 & 0.09 & 1043366.16 & $518.643^{*}$ & 0.96 & 0.87 & 13316.65 & 412.970 \\
\hline$t_{1(3)}^{r}$ & 0.59 & 76.49 & 95308.27 & 885.285 & 0.74 & 0.09 & 1043366.29 & $518.643^{*}$ & 0.96 & 0.98 & 13316.98 & 412.960 \\
\hline$t_{1(4)}^{r}$ & 0.60 & 76.71 & 95468.42 & 883.800 & 0.74 & 0.09 & 1043369.73 & 518.641 & 0.96 & 3.10 & 13323.21 & 412.766 \\
\hline$t_{1(5)}^{r}$ & 0.60 & 76.59 & 95386.75 & 884.557 & 0.74 & 0.09 & 1043367.79 & 518.642 & 0.96 & 2.19 & 13320.59 & 412.848 \\
\hline$t_{1(6)}^{r}$ & 0.59 & 76.48 & 95303.94 & 885.325 & 0.74 & 0.09 & 1043366.14 & $518.643^{*}$ & 0.96 & 0.85 & 13316.58 & 412.972 \\
\hline$t_{1(7)}^{r}$ & 0.59 & 76.48 & 95304.34 & 885.322 & 0.74 & 0.09 & 1043366.03 & $518.643^{*}$ & 0.96 & 0.83 & 13316.52 & 412.974 \\
\hline$t_{1(8)}^{r}$ & 0.60 & 76.73 & 95485.97 & 883.638 & 0.75 & 0.09 & 1049814.73 & 515.457 & 0.96 & 4.25 & 13326.36 & 412.669 \\
\hline$t_{1(9)}^{r}$ & 0.59 & 76.48 & 95303.94 & 885.325 & 0.74 & 0.09 & 1043366.03 & $518.643^{*}$ & 0.96 & 0.81 & 13316.47 & 412.975 \\
\hline$t_{1(10)}^{r}$ & 0.59 & 76.47 & 95300.40 & $885.358^{*}$ & 0.74 & 0.09 & 1043366.03 & $518.643^{*}$ & 0.96 & 0.70 & 13316.13 & $412.986^{*}$ \\
\hline
\end{tabular}

* indicates the largest PRE

Table 6: Optimum values $\left(\psi_{1}^{*}, \psi_{2}^{*}\right)$, MSEs and PREs of different estimators $\left(t_{2(i)}^{r}, i=1\right.$ to 10) generated from proposed estimator $\mathrm{t}_{2}$.

\begin{tabular}{|c|c|c|c|c|c|c|c|c|c|c|c|c|}
\hline \multirow[b]{2}{*}{ Estimators } & \multicolumn{4}{|c|}{ Population 1} & \multicolumn{4}{|c|}{ Population 2} & \multicolumn{4}{|c|}{ Population 3} \\
\hline & $\psi_{1}^{*}$ & $\psi_{2}^{*}$ & MSE & PRE & $\psi_{1}^{*}$ & $\psi_{2}^{*}$ & MSE & PRE & $\psi_{1}^{*}$ & $\psi_{2}^{*}$ & MSE & PRE \\
\hline$t_{2(1)}^{r}$ & 0.53 & 3.98 & 45246.37 & 1864.791 & 0.50 & 1.57 & 202185.29 & 2676.430 & 0.94 & 0.13 & 12986.83 & 423.458 \\
\hline$t_{2(2)}^{r}$ & 0.53 & 3.98 & 44081.39 & 1914.073 & 0.50 & 1.57 & 202155.53 & 2676.824 & 0.94 & 0.09 & 13116.65 & 419.267 \\
\hline$t_{2(3)}^{r}$ & 0.53 & 3.98 & 44131.01 & 1911.921 & 0.50 & 1.57 & 202157.65 & 2676.796 & 0.94 & 0.10 & 13107.25 & 419.567 \\
\hline$t_{2(4)}^{r}$ & 0.53 & 3.98 & 46177.73 & 1827.179 & 0.50 & 1.57 & 202210.82 & 2676.092 & 0.94 & 0.15 & 12931.31 & 425.276 \\
\hline $\mathrm{t}_{2(5)}^{\mathrm{r}}$ & 0.53 & 3.98 & 45127.48 & 1869.703 & 0.50 & 1.57 & 202180.85 & 2676.489 & 0.94 & 0.12 & 13005.06 & 422.864 \\
\hline $\mathrm{t}_{2(6)}^{\mathrm{r}}$ & 0.53 & 3.98 & 44076.42 & 1914.289 & 0.50 & 1.57 & 202155.27 & 2676.828 & 0.94 & 0.09 & 13118.60 & 419.204 \\
\hline $\mathrm{t}_{2(7)}^{\mathrm{r}}$ & 0.53 & 3.98 & 44081.39 & 1914.073 & 0.50 & 1.57 & 202155.53 & 2676.824 & 0.94 & 0.09 & 13116.65 & 419.267 \\
\hline$t_{2(8)}^{r}$ & 0.53 & 3.98 & 46405.23 & 1818.222 & 0.54 & 1.52 & 297759.98 & 1817.352 & 0.94 & 0.17 & 12844.01 & $428.167^{*}$ \\
\hline$t_{2(9)}^{r}$ & 0.53 & 3.98 & 44076.42 & 1914.289 & 0.50 & 1.57 & 202153.61 & $2676.850^{*}$ & 0.94 & 0.09 & 13121.61 & 419.108 \\
\hline $\mathrm{t}_{2(10)}^{\mathrm{r}}$ & 0.53 & 3.98 & 44031.68 & $1916.234^{*}$ & 0.50 & 1.57 & 202153.61 & $2976.850^{*}$ & 0.94 & 0.09 & 13131.21 & 418.802 \\
\hline
\end{tabular}

* indicates the largest PRE 
It is observed from Table 4 that the regression estimator $t_{\text {Reg }}$ and Kadilar and Cingi's $\left(2006\right.$ a) estimatorst ${ }_{\mathrm{KCj}}^{* *}(j=2,3,4,5)$ at the optimum condition, are more efficient than the usual unbiased estimator $t_{0}$, ratio estimator $t_{R}$, Sisodia and Dwivedi's (1981) estimator $t_{S D}$, Upadhyaya and Singh's (1999) estimators $t_{\text {US1 }}$, $\mathrm{t}_{\mathrm{us} 2}$; Singh and Tailor's (2003) estimator $\mathrm{t}_{\mathrm{sT}}$, Singh's (2003) estimators $\mathrm{t}_{\mathrm{s} 1}, \mathrm{t}_{\mathrm{s} 2}$; Singh et al.'s (2004) estimator $t_{\text {STK }}$, Kadilar and Cingi's (2004) estimators $t_{\text {KCi }}$ $(i=1,2,3,4,5)$, Kadilar and Cingi's (2006 b) estimators $t_{K i}(i=1,2,3,4)$ and Kadilar and Cingi's $(2006 \mathrm{c})$ estimators $t_{\mathrm{KCi}}^{*}(i=1,2,3,4,5)$ for all three populations.

Table 5 clearly shows that the minimum MSEs of the estimators $t_{1(j)}^{r}(j=1$ to 10) depend on the transformations used. The estimators $t_{1(6)}^{r}$ and $t_{1(9)}^{r}$ have smallest MSE (at optimum conditions) among all the estimators $t_{1(j)}^{r}(j=1$ to 9$)$ and largest PRE for population I. However the estimators $t_{1(\mathrm{j})}^{r}(j=1$ to 10$)$ except the estimator $\mathrm{t}_{1(8)}^{\mathrm{r}}$ are almost equally efficient for all three populations I, II and III. It is further observed from Table 4 and Table 5 that there is larger gain in efficiency by using the estimator $t_{1(\mathrm{j})}^{r}(j=1$ to 10$)$, (which are members of Gupta and Shabbir (2008) estimator $t_{1}$ ) over regression estimator $t_{\text {Reg }}$ and Kadilar and Cingi's (2006a) estimator $t_{K C j}^{* *}(j=2,3,4,5)$. We also note that the estimator $t_{1(10)}^{r}$ (based only on the population mean $\bar{X}$ ) has smaller MSE (at optimum condition) among the estimators $t_{1(\mathrm{j})}^{r}(j=1$ to 10$)$ for population I and III while for population II the MSEs (at optimum condition) of the estimators $t_{1(7)}^{r}, t_{1(9)}^{r}$ and $t_{1(10)}^{r}$ are same.

It is observed from Table 6 that the estimator $t_{2(10)}^{r}$ has largest PRE for population I. The estimators $t_{2(9)}^{r}$ and $t_{2(10)}^{r}$ are equally efficient but have largest efficiency among allthe estimator in population II. In population III the estimator $t_{2(8)}^{r}$ has the largest efficiency among all the estimators $t_{2(j)}^{r}(j=1$ to 10).

Finally we conclude that the estimators $t_{2(j)}^{r}(j=1$ to 10) (generated from the proposed class of estimators $t_{2}$ ) are more efficient than the estimator $t_{0}, t_{R}, t_{S D}$ , $t_{\text {US1 }}, t_{\text {US2 }}, t_{S T}, t_{S 1}, t_{S 2}, t_{S T K}, t_{K C i}^{*}(i=1$ to 5$), t_{K i}$ ( $i=1$ to 4$), t_{K C i}^{*}(i=1$ to 5$)$, $t_{K C j}^{* *}(j=2,3,4,5)$, the regression estimator $t_{\text {Reg }}$ and the estimators $t_{1(j)}^{r}(j=1$ to 10) which are generated from Gupta and Shabbir (2008) class of estimators $t_{1}$. 
There is no significant role of transformations used in Gupta and Shabbir (2008) estimator $t_{1}$ and the proposed class of estimators $t_{2}$ as the estimator $t_{1(10)}^{r}$ and $t_{2(10)}^{r}$ (which are only based on population mean $\bar{X}$ of the auxiliary variable $x$ ) appears to the best estimator in the sense of having largest efficiency for all three populations.

However this conclusion should not be extrapolated in general. There may be possibility of populations in practical situations where the transformations used in the estimators $t_{1}$ and $t_{2}$ may play significant role. Thus based on the above discussions we recommend the estimators $t_{2(j)}^{r}(j=1$ to 10) generated from the proposed class of estimators $t_{2}$ for their use in practice.

\section{Conclusion}

A revisit to the Gupta and Shabbir (2008) estimator $t_{1}$ has been made in this paper. We have derived the correct MSE expression of Gupta and Shabbir (2008) estimator $t_{1}$. The correct MSE expression depend upon the transformation used, so different estimators $t_{1(j)}^{r}(j=1$ to 9) give the different minimum MSEs and hence PREs. Similar is the case with the estimators $t_{2(j)}^{r}(j=$ 1 to 9 ) generated from the proposed class of estimators $t_{2}$. Theoretically and empirically it has been shown that the estimators $t_{1}$ and $t_{2}$ (at their optimum conditions) are better than usual regression estimator $t_{\text {Reg }}$ and other competing estimators considered here. Finally with help of the three numerical data we have shown that the estimators $t_{2(j)}^{r}(j=1$ to 10$)$ generated from proposed class of estimators $t_{2}$ are more efficient than the estimators $t_{1(j)}^{r}(j=1$ to 10) generated from Gupta and Shabbir (2008) estimator $t_{1}$, usual regression estimator $t_{\text {Reg }}$ and other competing estimators considered here. Thus our recommendation goes in the favor of proposed class of estimators $t_{2}$.

\section{Acknowledgement}

The authors are deeply grateful to the Editor Dr. Shahid Kamal and to the referees for their helpful comments and valued suggestions that led to this improved version of the paper.

\section{References}

1. Ahmed, M.S., Roman, M.S. and Hossain, M.I. (2000). Some competitive estimators of finite population variance using multivariate auxiliary information. Infor. Manage. Scie., 11(1), 49-54. 
2. Ahmed, M.S., Walid, A.D. and Ahmed, A.O.H. (2003). Some estimators for finite population variance under two-phase sampling. Statist. Trans., 6(1), 143-150.

3. Das, A.K. and Tripathi, T.P. (1978). Use of auxiliary information in estimating the finite population variance. Sankhya, C, 40, 139-148.

4. Gupta, S. and Shabbir, J. (2008). On improvement in estimating the population mean in simple random sampling. Jour. Appl. Statist., 35(5), 559-566.

5. Jhajj, H.S., Sharma, M.K. and Grover, L.K. (2005). An efficient class of chain estimators of population variance under sub-sampling. Jour. Jap. Statist. Soc., 35(2), 273-286.

6. Jhajj, H.S., Sharma, M.K. and Grover, L.K. (2006). Dual to ratio estimators of finite population mean obtained on using linear transformation to auxiliary variable. Jour. Japan Statist. Soc. 36(1) 107-119.

7. Kadilar, C. and Cingi, H. (2004). Ratio estimators in simple random sampling. Appl. Math. Comput., 151 (3), 893-902.

8. Kadilar, C. and Cingi, H. (2006a). Improvement in estimating population mean in simple random sampling. Appl. Math. Lett., 19(1), 75-79.

9. Kadilar, C. and Cingi, H. (2006b). An improvement in estimating population mean using correlation coefficient.Hacet. Jour. Math. Statist., 35(1), 103-109.

10. Kadilar, C. and Cingi, H. (2006c). New ratio estimators using correlation coefficient. Interstat, 4, 111-115.

11. Lui, K.J. (1990). Modified product estimators of finite population mean in finite sampling. Commun. Statist. Theo. Metho., 19(10), 3799-3807.

12. Prasad, B. (1989). Some improved ratio-type estimators of population mean and ratio in finite population sample surveys. Commun. Statist. Theo. Metho., 18, 379-392.

13. Prasad, B. and Singh, H.P. (1990). Some improved ratio-type estimators of finite population variance in sample surveys. Commun. Statist. Theo. Metho., 19(3), 1127-1139.

14. Prasad, B. and Singh, H.P. (1992). Unbiased estimators of finite population variance using auxiliary information in sample surveys. Commun. Statist. Theo. Metho., 21(5), 1367-1376.

15. Ray, S.K. and Sahai, A. (1980). Efficient families of ratio and product type estimators. Biometrika, 67, 211-215.

16. Reddy, V.N. (1973). On ratio and product methods of estimation. Sankhya, B, 35, 307-316.

17. Reddy, V.N. (1974). On a transformed ratio method of estimation. Sankhya, C, 36, 59-70.

18. Sahai, A. and Ray, S.K. (1980). An efficient estimator using auxiliary information. Metrika, 27, 271-275.

19. Singh, G.N. (2003). On the improvement of product method of estimation in sample surveys.Jour.Ind. Soc. Agri. Statist., 56(3), 267-275. 
20. Singh, H.P. (1986). A generalized class of estimators of ratio, product and mean using supplementary information on an auxiliary character in PPSWR sampling scheme. Guj. Statist. Rev., 13(2), 1-30.

21. Singh, H.P., and Agnihotri, N. (2008). A general procedure of estimating population mean using auxiliary information in sample surveys. Statist. Trans., 8(1), 71-87.

22. Singh, H.P., and Espejo, M.R. (2003). On linear regression and ratioproduct estimation of a finite population mean. The Statistician, 52(1), 59-67.

23. Singh, H.P. and Espejo, M.R. (2007). Double sampling ratio-product estimator of a finite population mean in sample surveys. Jour. Appl. Statist., 34(1), 71-85.

24. Singh, H.P. and Tailor, R. (2003). Use of known correlation coefficient in estimating the finite population mean. Statist. Trans., 6, 555-560.

25. Singh, H.P. and Vishwakarma, G.K. (2006). An efficient variant of the product and ratio estimators in stratified sampling. Statist. Trans., 7(6), 1311-1325.

26. Singh, H.P., Tailor, R., Singh, S. and Kim, J.M. (2008). A modified estimator of population mean power transformation. Statistical Papers, 49, 37-58.

27. Singh, H.P., Tailor, Rajesh, Tailor, Ritesh and Kakran, M.S. (2004). An improved estimator of population mean using power transformation.Jour.Ind. Soc. Agri. Statist., 58(2), 223-230.

28. Singh, R.K. and Singh, G. (1984). A class of estimators with estimated optimum values in sample surveys. Statist. Probab. Lett., 2, 319-321.

29. Sisodia, B.V.S., and Dwivedi,V.K. (1981). A modified ratio estimator using coefficient of an auxiliary variable.Jour.Ind. Soc. Agri. Statist., 33, 13-18.

30. Srivastava, S.K., and Jhajj, H.S. (1980). A class of estimators using auxiliary information for estimating finite population variance. Sankhya, C, 42, 87-96.

31. Srivastava, S.K., and Jhajj, H.S. (1983). A Class of estimators of the population mean using multi-auxiliary information. Cal. Stat. Assoc. Bull., 32, 47-56.

32. Tracy, D.S. and Singh, H.P. (1997).A family of estimators for population mean using prior/auxiliary information in sample surveys. Jour. Appl. Statist. Sci., 6(1), 1-20.

33. Tracy, D.S., Singh, H.P., and Singh, R. (1998). A class of almost unbiased estimators for finite population mean using two auxiliary variable. Biom. Jour., 40(6), 753-766.

34. Upadhyaya, L.N. andSingh, H.P. (1999). Use of transformed auxiliary variable in estimating the finite population mean. Biom. Jour., 41(5), 627-636. 